\title{
El cuadro de pastoreo en los sistemas extensivos de Patagonia Sur. Percepción y manejo de la heterogeneidad
}

\author{
Sebastián G. Ormaechea ${ }^{1, \otimes} ;$ Pablo L. Peri ${ }^{2,3,4} ;$ Pablo A. Cipriottit ${ }^{3,5}$ \& Roberto A. \\ DISTEL ${ }^{6,7}$ \\ ${ }^{1}$ EEA INTA Manfredi. Manfredi, Córdoba, Argentina. ${ }^{2}$ Universidad Nacional de la Patagonia Austral. ${ }^{3}$ Consejo Nacional \\ de Investigaciones Científicas y Técnicas (CONICET). ${ }^{4}$ EEA INTA Santa Cruz. ${ }^{5}$ Departamento de Métodos Cuantitativos \\ y Sistemas de Información, Facultad de Agronomía - IFEVA, Universidad de Buenos Aires. ${ }^{6}$ Departamento de Agronomía, \\ Universidad Nacional del Sur (CONICET). ${ }^{7}$ Centro de Recursos Naturales Renovables de la Zona Semiárida (CONICET).
}

\begin{abstract}
Resumen. La producción ganadera extensiva en la Patagonia Sur se realiza sobre cuadros de pastoreo de gran superficie, escasa receptividad animal y fragilidad ambiental elevada. El manejo histórico del pastoreo, desde fines del siglo XIX, causó la desertificación del paisaje en gran parte del territorio. Diferentes esfuerzos institucionales buscaron mejorar el manejo de los pastizales a través de adecuar la carga animal en los cuadros. No obstante, la heterogeneidad del paisaje dentro de éstos permite que los animales se concentren en los lugares que más prefieren. En este contexto, el desarrollo de recomendaciones técnicas para controlar el pastoreo heterogéneo requiere un conocimiento actualizado de la percepción de los productores frente a esta problemática. Por ello, se llevó adelante una encuesta a 82 productores ganaderos extensivos de Santa Cruz con el objetivo de caracterizar el manejo que realizan en los cuadros de pastoreo, los criterios para su diseño y su visión sobre el problema del pastoreo heterogéneo. Un porcentaje alto de productores $(\sim 75 \%)$ detecta la problemática, pero sólo $8.3 \%$ de ellos aplica más de 2 tecnologías para enfrentarla. En su conjunto, los productores muestran conocer una gran variedad de tecnologías. Sin embargo, los entrevistados señalaron diversas limitantes que determinan que las tecnologías no se consoliden como prácticas de uso común. El sistema extensivo de producción en la Patagonia Sur se sigue llevando adelante mayormente bajo un esquema de mínima intervención del paisaje, tal como en sus inicios. Es posible que sea necesario revisar el sistema de transferencia tecnológica desde las instituciones de investigación y extensión, pero también es imprescindible que el Estado participe diseñando e implementando políticas orientadas a proveer instrumentos idóneos para incentivar, promover, apoyar y monitorear la adopción de tecnología.
\end{abstract}

[Palabras clave: ovinos, ganadería, Santa Cruz, desertificación]

\begin{abstract}
Aвstract. The grazing paddock in the extensive systems of South Patagonia. Perception and management of heterogeneous grazing. The extensive livestock system of South Patagonia is carried out on large-scale paddocks, with low animal carrying capacity and high environmental fragility. The historical grazing management, since the end of the 19th century, has caused the desertification of the landscape in a large part of the territory. Different institutional efforts have sought to promote better management of rangelands based on adapting the stocking rate of the paddocks. However, the heterogeneity of the landscape within the paddocks still allows the concentration of animals in the most preferred places. In this context, the development of technical recommendations that allow the control of the heterogeneous grazing, requires an updated knowledge about the perception of the producers facing this problem. For this reason, a survey was carried out to 82 extensive livestock producers in Santa Cruz, seeking to characterize the management they perform in the paddocks, the criteria for their design and how they visualize the problem of heterogeneous grazing. A high percentage of producers ( $75 \%$ ) detects the problem, but only $8.3 \%$ applies more than 2 technologies to address it. The producers, as a whole, show knowledge of an important variety of technologies. However, different limitations indicated by the interviewees, determine that the technologies are not consolidated as common use practices. The extensive system in South Patagonia continues to be carried out mainly under a scheme of minimal intervention of the landscape, just as in its beginnings. It may be necessary to review the technology transfer system from research and extension institutions. However, the participation of the State is also essential, through the design and implementation of policies that provide suitable instruments to encourage, promote, support and monitor the adoption of technology.
\end{abstract}

[Keywords: sheep, animal husbandry, Santa Cruz, desertification]

Editor asociado: Alejandro Bisigato

ه ormaechea.sebastian@inta.gob.ar
Recibido: 6 de Octubre de 2018

Aceptado: 8 de Enero de 2019 


\section{INTRODUCCIÓN}

Hacia fines del siglo XIX, las tierras de la provincia de Santa Cruz fueron divididas en lotes cuadrangulares de 10000 ha, producto de un trazado geométrico sobre el territorio que no respondía a las características productivas de los suelos (Barbería 1995). Una segunda etapa del período de ocupación (19001914) contó con exploraciones previas que permitieron subdivisiones que tendían a lotes más relacionados con la receptividad ovina de los campos. Esto resultó en la definición de lotes rectangulares de 15000 a 20000 ha que contaban con diferentes proporciones de valles y mesetas (Barbería 1995). Estas subdivisiones catastrales son las que predominan hasta nuestros días, con algunas excepciones de zonas donde se conformaron subdivisiones menores con lotes cuadrangulares de 2500 ha o los situados en bordes costeros de forma irregular.

Los lotes, devenidos en establecimientos, se subdividieron internamente bajo un patrón común entre los ganaderos. Las divisiones del terreno se realizaron según la época de uso, en campos de veranada (comúnmente, en sitios de mayor altitud) e invernada (Martinic 1985). Luego, cada campo fue apotrerado por razones de manejo pecuario. Sin embargo, esa división se realizó a base de principios geométricos, con abstracción de las características ecológicas; primó la actividad de lucro económico al considerar sólo a la hacienda y nunca al suelo (Martinic 1985). El criterio generalizado fue construir rectángulos que seguían las líneas de paralelos y meridianos, sin considerar el componente pastizal (Suárez 2009). Como una consecuencia de ello, por lo general se observan sitios contrastantes (e.g., vegas, estepa arbustiva o pastizal) dentro de una misma unidad de manejo (cuadro de pastoreo), lo cual promueve el uso heterogéneo del pastizal al combinar sitios de pastoreo con diferente grado de atracción para el ganado (Golluscio et al. 1999; Cibils and Coughenour 2001; Ormaechea and Peri 2015). En este sentido, un estudio realizado con ovinos en arbustales del monte patagónico demuestra que las variaciones de cobertura de especies preferidas, la presencia de defensas estructurales o químicas anti-herbívoro en la vegetación y la obstaculización visual pueden determinar comportamientos de selección o aversión por determinados sitios del pastizal natural (Bertiller and Ares 2008). Por otra parte, la enorme superficie de los cuadros también resulta clave ya que, a mayor tamaño, la distribución de los herbívoros empeora, al igual que sus efectos espaciales en el largo plazo; así aumentan las diferencias entre las áreas más impactadas y las más evitadas (Oñatibia et al. 2018). Por ende, el tamaño y la heterogeneidad de los cuadros dificultan el control de la intensidad y la época de pastoreo de los sitios más productivos, generalmente escasos en superficie respecto a la superficie total del cuadro, los cuales tienden a degradarse (Golluscio et al. 1998; Anchorena et al. 2001).

Aunque el comportamiento selectivo de los animales es común en diferentes ecosistemas, en sitios áridos y semiáridos puede ser una de las mayores causas de degradación del pastizal (Schlesinger et al. 1990; Fuls 1992). En este sentido, Golluscio et al. (1998) señalan que el pastoreo heterogéneo es uno de los tres factores de manejo relacionados con el impacto del pastoreo sobre los pastizales de la estepa patagónica, lo cual aumenta la intensidad y la frecuencia de defoliación de los pastos palatables en las áreas preferidas. Esto lleva a la pérdida de vigor y a la muerte de plantas individuales.

El pastoreo año redondo o continuo es el tipo de manejo mayoritario en Patagonia Sur (Soriano 1983; Golluscio et al. 1999). Cada cuadro tiene asignado un número de animales y el manejo apunta a mantener ese número lo más estable posible. Esto se realiza con relativa independencia de la variabilidad de la oferta de forraje ocasionada principalmente por las precipitaciones. En este contexto, el ajuste de la carga sobre la base de la evaluación de disponibilidad del pastizal es reconocido como un elemento fundamental en la producción sustentable de pastizales áridos y semiáridos (Golluscio et al. 1998; Rimoldi 2004; Oliva et al. 2012; Distel 2013). Para el caso de los ambientes de estepa y matorrales (>90\% de la superficie de Santa Cruz), el Instituto Nacional de Tecnología Agropecuaria (INTA) desarrolló un método de evaluación de pastizales muy recomendado en el sector productivo (Borrelli and Oliva 2001). Sin embargo, su grado de adopción como práctica frecuente no supera el $17 \%$ de los establecimientos ganaderos extensivos en gran parte de la provincia (Ormaechea et al. 2009; Sturzenbaum 2012). Todas estas condiciones, en conjunto con una carga animal excesiva de los cuadros de pastoreo (Consorcio DHV 1999; Aagesen 2000; Cibils and Coughenour 2001) determinó una degradación moderada a severa de casi el $75 \%$ de los suelos de Santa Cruz (del Valle 1998). 
Los pastizales patagónicos son ecosistemas muy frágiles (entendiendo por fragilidad a la susceptibilidad que tienen estos ambientes de sufrir la pérdida de especies palatables o de receptividad forrajera a causa de un manejo inadecuado [Deregibus 1988; Oliva et al. 2001; Coronato 2015; Fariña et al. 2018]), y que la compatibilidad entre ganadería y conservación es difícil de alcanzar (Cingolani et al. 2008). Sin embargo, diferentes tecnologías pueden aportar a un uso más uniforme de los cuadros extensivos para así permitir su uso más eficiente y sustentable. La instalación de nuevas aguadas, el ajuste de la carga animal, el diseño de la forma y tamaño de los cuadros, la separación de ambientes, el pastoreo rotativo, el uso estratégico de bloques nutricionales, la combinación de categorías ovinas y los arreos o repuntes periódicos a sitios subutilizados son algunas de las estrategias con respaldo experimental en diferentes regiones del mundo (Williams 1954; Hart et al. 1993; Sevi et al. 2001; Tanaka et al. 2007; Holechek et al. 2010; Bohnert and Stephenson 2016; di Virgilio and Morales 2016). Ante este panorama, la percepción del productor sobre el impacto de los animales en el pastizal natural es uno de los elementos básicos a considerar para avanzar en recomendaciones técnicas al sector. Por ello, el objetivo de este trabajo fue realizar un diagnóstico de los criterios que actualmente aplica el sector ganadero para diseñar y manejar cuadros extensivos en la Patagonia Sur, poniendo un énfasis especial en cómo el productor percibe el pastoreo heterogéneo.

\section{MATERIALES y MÉTOdOS}

La información se relevó por medio de una encuesta a productores o encargados de estancias de la provincia de Santa Cruz. La población a muestrear incluyó a todos los establecimientos con ganadería extensiva en el territorio provincial, y se excluyó a aquellos con superficies menores a 500 ha, reservas naturales, terrenos fiscales y establecimientos con menos de 100 ovinos o 10 vacunos. Para determinar el número total de establecimientos se utilizó información ganadera provista por SENASA (período 2016) y datos de superficie obtenidos del catastro provincial (González and Rial 2006). Se relevó un total de 82 establecimientos, lo que representa $12.2 \%$ de la población muestreada (670 explotaciones ganaderas). La identificación de los establecimientos relevados y su localización geográfica se realizó con información GIS del catastro provincial de Santa Cruz. El muestreo fue de tipo no aleatorio, ya que la elección de los establecimientos estuvo supeditada a la facilidad de contacto con los entrevistados. Sin embargo, con el objetivo de buscar la representatividad de toda la extensión provincial se recurrió a la colaboración de agencias de extensión rural de INTA y a técnicos del medio rural de todo el territorio. Con el mismo objetivo, la encuesta también se realizó en el marco de diferentes eventos rurales provinciales a fin de contactar a aquellos productores no vinculados de forma directa con el INTA. Las encuestas fueron realizadas personalmente y siempre por el mismo encuestador para evitar diferentes apreciaciones en las particularidades de cada pregunta (Alaminos and Castejón 2006).

La encuesta contó con cinco preguntas de múltiple opción de carácter cerrado y 12 preguntas de múltiple opción de carácter semiabierto al agregar la opción "otra" e invitar a especificar el detalle de la respuesta (Apéndice 1). Además, a lo largo de la encuesta se incluyeron cuatro espacios para comentarios o aclaraciones de respuestas a fin de detectar aspectos no considerados inicialmente en la consulta. Al formular las preguntas se priorizó el objetivo de facilitar la comprensión por parte del productor; para ellos se escogieron preguntas cortas, sencillas, sin ambigüedades ni palabras cargadas emocionalmente (Alaminos and Castejón 2006). No se pudieron evitar preguntas que recurran a la memoria y a cálculos matemáticos. Sin embargo, en estos casos se consultó de manera general y sobre datos promedio de alta familiaridad para un encargado de estancia (Anguita et al. 2003). Se tuvo especial atención en proveer al encuestado la identificación del organismo que llevó a cabo la investigación, en explicar la importancia del estudio y en establecer de manera explícita un compromiso de devolución de la información generada. Cabe aclarar, que, como medida precautoria, previamente se realizó una prueba piloto sobre cinco productores a fin de evaluar si las preguntas se comprendían bien y no producían fatiga ni rechazo al entrevistado (Anguita et al. 2003).

En la pregunta 1 se consultó sobre el tipo de manejodelpastoreo.Seofrecieron5alternativas con sus respectivas interpretaciones: Año redondo (los animales permanecen todo el año en el mismo cuadro), Estacional Continuo (los cuadros se usan en períodos menores a un año y siempre en la misma época (e.g., veranadainvernada, veranada-invernada-parición), 
Estacional Rotativo (los cuadros se usan en períodos menores a un año y la época de uso cambia entre años), Rotativo (los cuadros se usan más de una vez al año como resultado de una planificación del pastoreo; si un cuadro se usara más de una vez al año por escasez de forraje, no se considera rotativo) y Otros tipos de manejo. Para formular la pregunta 6 (acerca de la distancia predominante entre fuentes de agua permanente) fue necesario establecer rangos de distancia que caractericen de manera sencilla el manejo por parte del productor. De acuerdo a Holechek et al. (2010), los ovinos se distancian del agua entre 1.6 a 6.9 km según la raza y la categoría. Por su parte, Squires et al. (1972) destaca que, a partir de $4 \mathrm{~km}$, los ovinos toman agua sólo una vez por día, lo cual condiciona su desempeño productivo. Teniendo en cuenta esta información se establecieron 3 rangos (menos de $1.5 \mathrm{~km}$; entre 1.5 y $4 \mathrm{~km}$; más de $4 \mathrm{~km}$ ) que representaron un compromiso entre lo encontrado por estos autores y la búsqueda de claridad en la formulación de las preguntas. No obstante, estos rangos no permiten predecir un uso más o menos uniforme del pastizal, ya que las distancias óptimas entre fuentes de agua son una particularidad de cada ambiente y se los debe analizar según las características de cada sitio de interés (Bailey et al. 1996).

Sobre la base de toda la información obtenida se realizó un análisis estadístico descriptivo, para lo que se calculó el porcentaje de establecimientos que respondían a una característica específica sobre el total de establecimientos. Asimismo, se realizó una clasificación de productores según su percepción y acción referente al problema del pastoreo heterogéneo. Para dicha clasificación, primero se utilizó la pregunta 9 para diferenciar entre productores que detectan o no el pastoreo desparejo (i.e., heterogéneo). Luego se tomó la pregunta 10 para definir qué productores accionan frente a la problemática con la aplicación o no de estrategias para lograr un uso parejo por parte de los animales. Finalmente, a partir de la pregunta 11 se determinó cuáles productores no aplican estrategias, cuáles lo hacen por desconocer alternativas de remediación y cuáles lo hacen por limitaciones operativas o económicas. Por otro lado, se revisaron todas las aclaraciones y apreciaciones expuestas en los espacios brindados para comentarios, con la intención de identificar mensajes comunes y relevantes para analizar la problemática.

\section{Resultados}

La provincia de Santa Cruz cuenta con 670 establecimientos con ganadería extensiva, cuya superficie media es 21145 ha. El 55.2\% posee exclusivamente ganadería ovina, mientras que $29.2 \%$ tiene producción mixta ovino-bovino. El 15.6\% restante se dedica a la producción bovina.

En su gran mayoría (74.6\%), los productores reconocieron la problemática del pastoreo heterogéneo (Figura 1). A su vez, un 41.5\% del total de productores aplican estrategias para atender esta problemática. Sin embargo, sólo $8.3 \%$ de los productores aplica más de 2

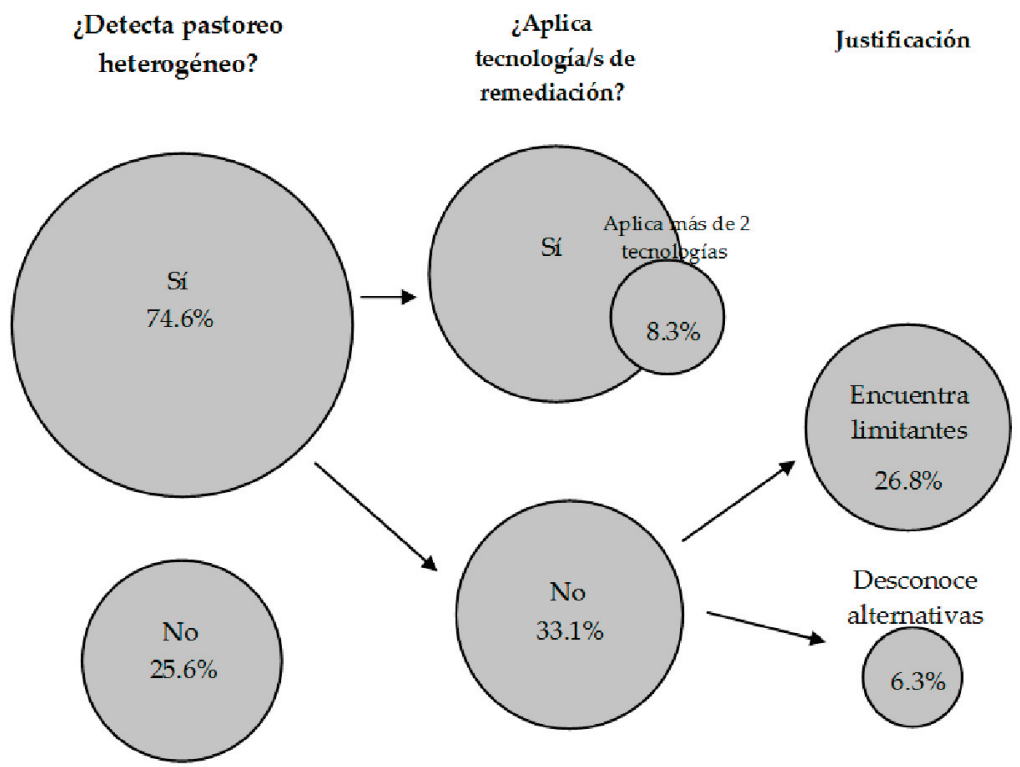

Figura 1. Clasificación de productores según su percepción y acción sobre el problema del pastoreo heterogéneo. Los porcentajes expuestos fueron calculados sobre el total de encuestados en todos los casos. El tamaño de los círculos es proporcional al porcentaje expuesto.

Figure 1. Landholder's classification according to their perception and action on the problem of heterogeneous grazing. The percentages exposed were calculated on the total number of respondents in all cases. The size of the circles is proportional to the percentage exposed. 
tecnologías de remediación. Por ende, el 91.7\% de los productores encuestados no aplica un manejo integral o más de dos tecnologías para abordar la problemática del pastoreo heterogéneo. El 33.1\% de los consultados plantearon desconocer alternativas tecnológicas o encontrar diferentes tipos de limitantes.

Se destacó un porcentaje elevado de manejo del pastoreo tipo continuo (78\%), representado por las modalidades Año redondo y Estacional Continuo (Tabla 1). El número promedio de cuadros por establecimiento apenas supera las 10 unidades, y sólo 9.1\% delos establecimientos posee más de 20 cuadros. Las superficies de los cuadros se distribuyeron de manera equitativa entre las diferentes categorías de superficie establecidas, y se destaca la existencia de numerosos cuadros de más de 2500 ha (Tabla 1). El distanciamiento entre aguadas es, en su mayoría, entre 1.5 a 4 km, y están dispuestas en

Tabla 1. Manejo del pastoreo, características y criterios de diseño de los cuadros en establecimientos ganaderos extensivos de Santa Cruz, Argentina.

Table 1. Grazing management, characteristics and design criteria of the paddocks in extensive livestock ranches of Santa Cruz, Argentina.

\begin{tabular}{|c|c|c|}
\hline Tipo de manejo del pastoreo & $\begin{array}{l}\text { Año redondo } \\
\text { Estacional Continuo } \\
\text { Estacional Rotativo } \\
\text { Rotativo } \\
\text { Otros }\end{array}$ & $\begin{array}{l}45.1 \% \\
32.9 \% \\
12.2 \% \\
3.7 \% \\
6.1 \%\end{array}$ \\
\hline $\begin{array}{l}\text { Porcentaje de establecimientos } \\
\text { que tienen al menos un cuadro } \\
\text { dentro de las siguientes } \\
\text { categorías }^{1}\end{array}$ & $\begin{array}{l}\text { Menos de } 1000 \text { ha } \\
1000 \text { a } 2499 \text { ha } \\
2500 \text { a } 5000 \text { ha } \\
\text { Más de } 5000 \text { ha }\end{array}$ & $\begin{array}{l}40.8 \% \\
55.3 \% \\
68.4 \% \\
52.6 \%\end{array}$ \\
\hline Número promedio de cuadros po & r establecimiento \pm desvío estándar & $10.5 \pm 8.6$ \\
\hline $\begin{array}{l}\text { Categoría de número de cuadros } \\
\text { por establecimiento }\end{array}$ & $\begin{array}{l}\text { Menos de } 10 \\
10 \text { a } 20 \\
\text { Más de } 20\end{array}$ & $\begin{array}{c}67.5 \% \\
23.4 \% \\
9.1 \%\end{array}$ \\
\hline $\begin{array}{l}\text { Disposición predominante de las } \\
\text { fuentes de agua en los cuadros }\end{array}$ & $\begin{array}{l}\text { Esquineros } \\
\text { Laterales } \\
\text { Otro lugar }\end{array}$ & $\begin{array}{c}3.7 \% \\
11.1 \% \\
85.2 \%\end{array}$ \\
\hline $\begin{array}{l}\text { Distancia predominante entre } \\
\text { aguadas }\end{array}$ & $\begin{array}{l}\text { Menor a } 1.5 \mathrm{~km} \\
\text { Entre } 1.5 \mathrm{y} 4 \mathrm{~km} \\
\text { Más de } 4 \mathrm{~km} \\
\text { Desconoce o no recuerda }\end{array}$ & $\begin{array}{c}27.5 \% \\
57.5 \% \\
10 \% \\
5 \%\end{array}$ \\
\hline $\begin{array}{l}\text { Superficie de pastoreo por } \\
\text { aguada }\end{array}$ & $\begin{array}{l}\text { Menos de } 200 \text { ha } \\
\text { Entre } 200 \text { y } 1600 \text { ha } \\
\text { Más de } 1600 \text { ha } \\
\text { Desconoce o no recuerda }\end{array}$ & $\begin{array}{c}4.9 \% \\
63 \% \\
14.8 \% \\
17.3 \%\end{array}$ \\
\hline $\begin{array}{l}\text { Porcentaje de productores que } \\
\text { señalaron los siguientes criterios } \\
\text { como utilizados originalmente } \\
\text { para el diseño de los cuadros }{ }^{1}\end{array}$ & $\begin{array}{l}\text { Localización de fuentes de agua naturales o posibilidad de realizar pozos } \\
\text { Facilidades para el arreo y movimiento de animales. } \\
\text { Separación de sitios de mayor preferencia por ovinos } \\
\text { Separación ambientes o tipos de vegetación } \\
\text { Desconoce o no recuerda } \\
\text { Otros }\end{array}$ & $\begin{array}{c}36.6 \% \\
12.2 \% \\
8.5 \% \\
7.3 \% \\
53.6 \% \\
13.2 \%\end{array}$ \\
\hline $\begin{array}{l}\text { Porcentaje de productores } \\
\text { que actualmente poseen los } \\
\text { siguientes criterios para el diseño } \\
\text { de los cuadros }{ }^{1}\end{array}$ & $\begin{array}{l}\text { Localización de fuentes de agua naturales o posibilidad de realizar pozos } \\
\text { Facilidades para el arreo y movimiento de animales } \\
\text { Separación sitios de mayor preferencia por ovinos } \\
\text { Separación ambientes o tipos de vegetación } \\
\text { Predadores (amenaza de zorros, pumas y/o perros) } \\
\text { Logro de pastoreo parejo del cuadro } \\
\text { Incremento de subdivisiones para mejorar el manejo } \\
\text { Abigeato } \\
\text { Otros }\end{array}$ & $\begin{array}{c}53.8 \% \\
28.7 \% \\
22.5 \% \\
35 \% \\
10 \% \\
25 \% \\
52.5 \% \\
22.5 \% \\
10.4 \%\end{array}$ \\
\hline
\end{tabular}

${ }^{1}$ En estos casos, la suma de porcentajes no necesariamente es igual al 100\%, ya que el entrevistado tuvo habilitada la selección de más de una opción 
diferentes sitios de los cuadros. La superficie de pastoreo predominante por aguada (63\%) es entre 200 y 1600 ha. Entre los criterios que se tuvieron originalmente para el diseño de los cuadros, predominó la localización de aguadas naturales o la posibilidad de realizar pozos (Tabla 1). Sin embargo, gran parte de los productores $(53.6 \%$ ) desconoce o no recuerda los criterios que se usaron. Entre los criterios actuales para el diseño de los cuadros también predominó la localización de fuentes de agua naturales o la posibilidad de realizar pozos (53.8\%). Sin embargo, surgieron nuevos criterios con altos porcentajes de incidencia tales como "lograr un pastoreo parejo del cuadro" $(25 \%)$ y la intención de realizar mayores subdivisiones a fin de mejorar el manejo del pastoreo (52.5\%).

Al relevar la percepción de los productores y encargados sobre el pastoreo heterogéneo, predominó la consideración de un pastoreo no uniforme de los cuadros con sectores sobrepastoreados y subutilizados (Tabla 2). Los encuestados señalan diferentes factores causantes de esta situación, pero predominan algunos tales como la falta de fuentes de agua y la predisposición de los animales al uso de sitios preferidos.

Casi $60 \%$ de los encuestados considera que el pastoreo heterogéneo no es un problema

Tabla 2. Consideraciones sobre la percepción y el manejo del pastoreo heterogéneo en establecimientos ganaderos extensivos de Santa Cruz, Argentina.

Table 2. Considerations on the perception and management of heterogeneous grazing in extensive livestock ranches in Santa Cruz, Argentina.

\begin{tabular}{|c|c|c|}
\hline $\begin{array}{l}\text { Apreciación sobre el uso de los } \\
\text { cuadros extensivos por parte de } \\
\text { los animales }\end{array}$ & $\begin{array}{l}\text { Homogéneo } \\
\text { Heterogéneo con sectores sobrepastoreados } \\
\text { Heterogéneo con sectores subutilizados } \\
\text { Heterogéneo con ambas situaciones } \\
\text { Otro }\end{array}$ & $\begin{array}{c}23.2 \% \\
25.6 \% \\
11 \% \\
36.6 \% \\
3.6 \%\end{array}$ \\
\hline $\begin{array}{l}\text { Porcentaje de productores que } \\
\text { señalan los siguientes factores } \\
\text { como causantes del pastoreo } \\
\text { heterogéneo }{ }^{1,2}\end{array}$ & $\begin{array}{l}\text { Falta de fuentes de agua } \\
\text { Sitios preferidos } \\
\text { Influencia del viento } \\
\text { Tamaño de los cuadros } \\
\text { Predadores (amenaza de zorros, pumas y/o perros) } \\
\text { Desconoce } \\
\text { Otros }\end{array}$ & $\begin{array}{c}48.4 \% \\
51.6 \% \\
30.6 \% \\
30.6 \% \\
8.1 \% \\
1.6 \% \\
16 \%\end{array}$ \\
\hline $\begin{array}{l}\text { Percepción del grado de } \\
\text { importancia del pastoreo } \\
\text { heterogéneo }{ }^{2}\end{array}$ & $\begin{array}{l}\text { No es un problema } \\
\text { Afecta solo algunos sitios aislados } \\
\text { Afecta varios cuadros, pero no requiere atención inmediata } \\
\text { Afecta varios cuadros y requiere atención inmediata } \\
\text { Es uno de los problemas más importantes del establecimiento }\end{array}$ & $\begin{array}{c}3.3 \% \\
42.6 \% \\
13.2 \% \\
31.1 \% \\
9.8 \%\end{array}$ \\
\hline $\begin{array}{l}\text { Porcentaje de productores } \\
\text { que aplican las siguientes } \\
\text { estrategias para evitar el pastoreo } \\
\text { heterogéneo }^{1}\end{array}$ & $\begin{array}{l}\text { Instala nuevas aguadas } \\
\text { Separación de sitios o ambientes } \\
\text { Subdivisión de cuadros } \\
\text { Movimientos (Repuntes) periódicos de hacienda dentro de los cuadros } \\
\text { Combina especies o categorías animales } \\
\text { Bloques nutricionales } \\
\text { Otras } \\
\text { No aplica ninguna }\end{array}$ & $\begin{array}{c}33.3 \% \\
8.6 \% \\
13.6 \% \\
17.3 \% \\
7.4 \% \\
2.5 \% \\
6.2 \% \\
45.7 \%\end{array}$ \\
\hline $\begin{array}{l}\text { Porcentaje de productores que } \\
\text { presenta las siguientes limitaciones } \\
\text { para el uso de estrategias }{ }^{1}\end{array}$ & $\begin{array}{l}\text { Costos } \\
\text { Dificultades operativas } \\
\text { Falta de tiempo } \\
\text { Falta de recurso humano capacitado } \\
\text { Otras prioridades } \\
\text { Desconocimiento de alternativas } \\
\text { Otros } \\
\text { No observa limitaciones }\end{array}$ & $\begin{array}{c}57.7 \% \\
11.5 \% \\
7.7 \% \\
19.2 \% \\
11.5 \% \\
11.5 \% \\
3.9 \% \\
17.9 \%\end{array}$ \\
\hline
\end{tabular}

${ }^{1}$ En estos casos, la suma de porcentajes no necesariamente es igual al $100 \%$, ya que el entrevistado tuvo habilitada la selección de más de una opción

${ }^{2}$ Sólo incluye productores que detectaron pastoreo heterogéneo

${ }^{3}$ Por ejemplo, presencia de cañadones que dificultan la instalación de alambrados 
importante ni una temática que requiera atención inmediata. Al ser consultados por la aplicación de estrategias para evitar el pastoreo heterogéneo, predomina la instalación de nuevas aguadas. Sin embargo, un $45.7 \%$ del total de productores consultados reconoce que no aplica ninguna estrategia. La limitante principal para la aplicación de medidas que eviten el pastoreo heterogéneo es el costo de implementación de estas estrategias. Sin embargo, productores y encargados también citaron una variedad de otras limitantes, como ser la falta de recursos humanos capacitados, el desconocimiento de alternativas y aspectos operativos (Tabla 2).

La encuesta también incluyó preguntas sobre el manejo del pastoreo con relación al uso uniforme de los cuadros (Tabla 3). En cuanto al manejo diferencial de mallines, la respuesta más común fue la reserva para el verano a fin de tener un recurso alimenticio engordar los animales. Sin embargo, muchos productores $(31.2 \%)$ no realizan un manejo diferencial de este ambiente. El uso de bloques nutricionales como atractivo para el pastoreo de sectores subutilizados fue escaso (4.9\%). En cuanto a la aplicación de la evaluación de pastizales, sólo un 23.2\% manifestó realizarla frecuentemente y un 53.6\% no lo aplica o sólo lo hizo una vez como requisito para la solicitud de financiamiento por la Ley Ovina Nacional. En cuanto al uso combinado de especies animales o categorías ovinas, la mayoría de los consultados combina vacunos con ovinos $\mathrm{u}$ ovejas madre con borregas. Sin embargo, esta acción pocas veces implica la intención de lograr un pastoreo parejo del cuadro.

En los espacios destinados a aclaraciones o comentarios se consignó una gran variedad de temas. Los más recurrentes fueron los relacionados con la problemática de la sequía y la presencia importante de guanacos en los cuadros de pastoreo. Otros problemas citados fueron los costos operativos elevados, los depredadores y el abigeato. También hubo expresiones recurrentes relacionadas con la intención de llevar adelante nuevas medidas de manejo como instalar alambrados, realizar perforaciones para obtener nuevas fuentes de agua o implementar esquemas de suplementación. A esto se suman otras, nombradas en menor medida, como implementar la evaluación de pastizales, el manejo de las cargas animales o la implementación de riego. En conjunto con las anteriores, estas aclaraciones y

Tabla 3. Aplicación de tecnologías de manejo del pastoreo en establecimientos ganaderos extensivos de Santa Cruz, Argentina.

Table 3. Application of grazing management technologies in extensive livestock ranches in Santa Cruz, Argentina.

\begin{tabular}{llc}
\hline $\begin{array}{l}\text { Porcentaje de productores } \\
\text { que realizan las siguientes }\end{array}$ & Reserva para engorde estival & $16.3 \%$ \\
medidas de manejo sobre & Reserva estival para recuperación de ovejas pre-servicio & $10 \%$ \\
mallines $^{1}$ & Reservan otoñal para flushing alimenticio pre-servicio & $2.5 \%$ \\
& Utilización predominante para vacunos & $6.3 \%$ \\
& Otros manejos & $10 \%$ \\
& No realiza manejo diferencial & $31.2 \%$ \\
& No posee mallines o son escasos & $40 \%$ \\
Utilización de bloques & Sí, para nutrición del animal & $34.1 \%$ \\
nutricionales & Sí, para que utilicen sectores subutilizados y para nutrición del animal & $4.9 \%$ \\
& No utiliza bloques nutricionales & $61 \%$ \\
Uso de la tecnología de & Sí, todos los años & $23.2 \%$ \\
evaluación o chequeo de & Sí, cada 2 años o más & $23.2 \%$ \\
pastizales & Sí, como requisito de Ley ovina & $19.5 \%$ \\
& No usa la tecnología & $34.1 \%$ \\
Porcentaje de productores & Vacunos y ovinos & $31.7 \%$ \\
que combinan especies & Oveja madre y borrega & $32.9 \%$ \\
animales o categorías ovinas & Oveja madre y borrego & $12.2 \%$ \\
en un mismo cuadro & Oveja madre y capón & $7.3 \%$ \\
& Oveja madre, capones y borregos & $6.1 \%$ \\
& Capones y borregos & $28 \%$ \\
& Otras combinaciones & $14.4 \%$ \\
\hline
\end{tabular}

${ }^{1}$ En estos casos, la suma de porcentajes no necesariamente es igual al 100\%, ya que el entrevistado tuvo habilitada la selección de más de una opción 
comentarios contribuyen a mejorar el manejo de los pastizales.

\section{DISCUSIÓN}

La información obtenida en este trabajo muestra que un porcentaje alto de productores ( $75 \%$ ) detecta la problemática del pastoreo heterogéneo. No obstante, la percepción general fue la de un interés moderado o escaso en la temática del pastoreo heterogéneo, relativizado por otras problemáticas que el productor considera más importantes o urgentes. Esto queda demostrado por la baja aplicación de tecnologías de remediación, que, además, no se aplican de forma integrada en la mayoría de los casos, lo que impide un tratamiento más efectivo de la problemática (Bailey 2004; Hunt et al. 2007; Barnes et al. 2008).

Los resultados del relevamiento demuestran, además, que el diseño de los cuadros no varió demasiado respecto de lo que existió originalmente, luegodelprocesodesubdivisión de las tierras. Los cuadros de más de 1000 ha siguen siendo predominantes, en coincidencia con otros relevamientos provinciales (Álvarez 2009; Andrade et al. 2010). En este sentido, Álvarez (2009) encontró, para el departamento de Magallanes, que hay pocas subdivisiones internas en los campos (existen cuadros de no menos de 1500 - 2000 ha e, incluso, de hasta 10000 ha sin divisiones) y que no se considera la separación de los diferentes ambientes. Del mismo modo, en el caso del presente estudio, el criterio de separación de ambientes dentro de los cuadros apenas supera el $22 \%$ de los casos y generalmente no se aplica a todos los cuadros del establecimiento. En este sentido, Suárez (2009) describe para Santa Cruz una ganadería de tipo totalmente extensiva, con escasez de instalaciones y manejo, desarrollada en grandes cuadros con visibles grados de deterioro en los sectores donde se suele concentrar la hacienda. Del mismo modo, Milicevic (2013) señala: "El sistema de producción tradicional se caracteriza por la cría extensiva sobre pastizales naturales, con baja a nula modificación del paisaje o de los recursos naturales". Sin embargo, según el presente estudio, los productores, observados grupalmente, muestran el uso de una variedad importante de tecnologías de manejo (Tablas 2 y 3) y mencionan la intención de incorporar mejoras. Pero en ningún caso estos elementos están hoy consolidados como prácticas comunes para la gran mayoría de los productores.

La aplicación escasa de tecnologías por parte de los productores encontrada en este estudio es un tema abarcado extensamente por diferentes autores en la Patagonia Sur (Andrade 2003; Quargnolo et al. 2007; Álvarez 2009; Suárez 2009; Sturzenbaum 2012). Estos trabajos señalan como causas el costo, las complicaciones que puede implicar, la escala de los establecimientos, la escasez de mano de obra especializada, el análisis de riesgo, las decisiones compartidas entre dueños o socios, o simplemente la preferencia por una temática en particular (e.g., la genética animal). En este trabajo, los costos fueron claramente la limitante más importante para aplicar tecnologías, lo cual se relacionaría con la poca rentabilidad actual de la producción ovina en Santa Cruz (Williams 2009).

En el caso del pastoreo heterogéneo, es muy posible que la falta de un conocimiento real de la problemática tenga un rol preponderante en la baja aplicación de tecnologías. Así como la mayoría de los productores no concibe que la desertificación sea producto del manejo histórico de los campos (Andrade 2002, 2003), es muy posible que no detecte la importancia real de propender a un pastoreo más uniforme de los pastizales. En este sentido, Andrade (2003) describió la percepción de los productores sobre la crisis del ecosistema y señala que "...algunos productores aceptan que el sobrepastoreo es uno de los factores que llevaron a la situación actual, pero al explicar los problemas de su campo remiten constantemente a los bajos precios de la lana, cuestiones climáticas y depredadores". Por otro lado, esto podría sugerir que la problemática del pastoreo heterogéneo se encuentre en una fase inicial de reconocimiento. Sin embargo, ya en el año 1955, Woolfolk señalaba para la Patagonia que la distribución del ganado en pastoreo representaba un serio problema y que se debía al enorme tamaño de los cuadros, a las pocas instalaciones de riego, a la falta de uso de sal o suplementos en el área de apacentamiento y a la tendencia de los ovinos a concentrarse en determinados sitios del paisaje. Asimismo, el desarrollo de tecnologías como la evaluación de pastizales a fines del siglo pasado (Borrelli et al. 1990) ya tomaban en consideración la importancia de identificar en el campo si el pastoreo por parte de los animales era homogéneo a lo largo de los cuadros. Por 
su parte, Golluscio et al. (1998) explican que muchos productores de diferentes biozonas de la Patagonia mejoraron la distribución de animales a través de la división de cuadros y del desarrollo de aguadas, planteando el pastoreo en diferentes sitios de acuerdo a su fenología. El presente relevamiento reveló un gran interés por aumentar la subdivisión de los campos en la Patagonia Sur ( 53\%). Esto, aunque se viene aplicando lentamente debido a limitantes económicas, describe un cambio particular en los criterios de los productores respecto del diseño original de los cuadros. Por ello, y a partir de lo relevado en el presente trabajo, postulamos que, al menos para tecnologías que tienen que ver con un manejo más eficiente y sustentable del pastoreo, las causas podrían estar muy asociadas a una cuestión de prioridades en el uso de recursos escasos, en especial dinero y tiempo. Frente a márgenes económicos reducidos, los productores deben decidir permanentemente sobre el uso prioritario de sus exiguos excedentes. Pero además del aspecto económico, deben decidir sobre una mayor inversión de tiempo a un sistema productivo que posiblemente se complejizará como resultado de la aplicación de estas tecnologías.

Por su parte, Sturzenbaum (2012) estudió, mediante un relevamiento en el sureste de la provincia de Santa Cruz, la adopción de tecnologías por parte de los productores. Ella encontró que los productores comprenden bien el concepto de tecnologías y conocen gran parte de las que difunde el INTA. Sin embargo, en el caso de la evaluación de pastizales, los productores no parecen comprender en profundidad los beneficios que se logran con un manejo sustentable de los pastizales naturales; en parte, ello explica por qué aún aplican esta tecnología de manera regular. Sumado a que se observa que los productores practican una gran variedad de tecnologías que no se consolidan como prácticas de uso común, esto da cuenta de la necesidad de revisar el sistema de transferencia tecnológica, lo cual ya fue reconocido por varios autores (Quargnolo et al. 2007; Andrade 2009; Sturzenbaum 2012). A modo de ejemplo, en la actualidad no existen informes o cartillas técnicas que compilen y detallen las prácticas plausibles de ser aplicadas en los campos de la Patagonia Sur para remediar el pastoreo heterogéneo. No obstante, es posible que la sola difusión o transferencia no alcance y que resulte estratégicamente imprescindible la participación del Estado para diseñar e implementar políticas que provean instrumentos idóneos para incentivar, promover, apoyar y monitorear de manera eficaz la adopción de tecnología.

Finalmente, cabe preguntarse cuáles son las 'otras problemáticas' que el productor considera más importantes o urgentes que el pastoreo heterogéneo, y si realmente lo son. La encuesta no consultó específicamente cuáles eran. Sin embargo, el análisis de las problemáticas de los sistemas ganaderos extensivos de la región ha sido abarcado en varios estudios (Álvarez 2009; Ormaechea et al. 2009; Suárez 2009; Iglesias et al. 2015). Estas problemáticas son muy variadas y obedecen, en muchos casos, a cuestiones locales o coyunturales (e.g., falta de mano de obra idónea, ataque de depredadores, baja productividad, instalaciones deterioradas, problemas sanitarios, alta densidad de guanacos, falta de perforaciones para agua de bebida animal, altos costos fijos, endeudamiento, dificultades para organizarse o asociarse entre productores, abigeato), pero también a problemas estructurales en los que el productor no puede intervenir (e.g., el bajo precio de lana o de la carne). En conclusión, existe un problema de percepción de la importancia de la problemática en muchos casos, aunque se encuentra entreverado con la negación del problema o con la imposibilidad de actuar debido principalmente a restricciones económicas.

\section{REFERENCIAS}

Aagesen, D. 2000. Crisis and conservation at the end of the world: sheep ranching in Argentine Patagonia. Environmental Conservation 27:208-215. https:/ / doi.org/10.1017/S0376892900000229

Alaminos, A., and J. L. Castejón. 2006. Elaboración, análisis e interpretación de encuestas, cuestionarios y escalas de opinión. Universidad de Alicante. Alcoy: Marfil. Pp. 119.

Álvarez, R. 2009. Situación actual y aportes al desarrollo del sector ganadero ovino-extensivo del Departamento Magallanes - Provincia de Santa Cruz. Trabajo integrador para optar al grado de Especialista en Desarrollo Rural. Escuela para Graduados Alberto Soriano, Universidad de Buenos Aires, Buenos Aires, Argentina. Pp. 106.

Anchorena, J., A. Cingolani, E. Livraghi, M. Collantes, and S. Stofella. 2001. Manejo del pastoreo de ovejas en Tierra del Fuego. CONICET-INTA, Buenos Aires, Argentina. Pp. 47.

Andrade, L. 2002. Territorio y ganadería en la Patagonia Argentina: desertificación y rentabilidad en la Meseta Central 
de Santa Cruz. Economía, Sociedad y Territorio 3:675-706. https://doi.org/10.22136/est002002341

Andrade, L. 2003. Sociología de la desertificación en la Patagonia Austral: los productores ovinos de la Meseta Central de Santa Cruz. Theomai Nro. 7.

Andrade, L., V. Bedacarratx, R. Álvarez, and G. Oliva. 2010. Otoño en la estepa: ambiente, ganadería y vínculos en la Patagonia Austral. La Colmena, Buenos Aires, Argentina. Pp. 306.

Anguita, J. C., J. R Labrador, J. D. Campos, J. Casas Anguita, J. Repullo Labrador, and J. Donado Campos. 2003. La encuesta como técnica de investigación. Elaboración de cuestionarios y tratamiento estadístico de los datos (I). Atención primaria 31:527-538. https:/ / doi.org/10.1016/S0212-6567(03)70728-8

Bailey, D. W., J. E. Gross, E. A. Laca, L. R. Rittenhouse, M. B. Coughenour, D. M. Swift, and P. L. Sims. 1996. Mechanisms that result in large herbivore grazing distribution patterns. Journal of Range Management 49:386-400. https://doi.org/ $10.2307 / 4002919$

Bailey, D. W. 2004. Management strategies for optimal grazing distribution and use of arid rangelands. Journal of Animal Science 82 E-Suppl:E147-153. https://doi.org/10.2527/2004.8213_supplE147x

Barbería, E. M. 1995. Los dueños de la tierra en la Patagonia austral, 1880-1920. Universidad federal de la Patagonia Austral, Río Gallegos, Argentina. Pp. 475.

Barnes, M. K., B. E. Norton, M. Maeno, and J. C. Malechek. 2008. Paddock Size and Stocking Density Affect Spatial Heterogeneity of Grazing. Rangeland Ecology and Management 6:380-388. https://doi.org/10.2111/06-155.1

Bertiller, M. B., and J. O. Ares. 2008. Sheep spatial grazing strategies at the arid Patagonian Monte, Argentina. Rangeland ecology and management 61:38-47. https://doi.org/10.2111/07-130.1

Bohnert, D. W., and M. B. Stephenson. 2016. Supplementation and sustainable grazing systems. Journal of Animal Science 94:15-25. https://doi.org/10.2527/jas.2016-0520

Borrelli, P., C. Baetti, C. Cheppi, and M. Iacomini. 1990. Una metodología para evaluar los Pastizales de Santa Cruz. Revista Argentina de Producción Animal. Suplemento 1:10-18.

Borrelli, P., and G. Oliva. 2001. Evaluación de pastizales. Pp. 163-184 in P. Borrelli and G. Oliva (eds.). Ganadería Ovina Extensiva Sustentable en la Patagonia Austral. Ediciones EEA INTA Santa Cruz, Río Gallegos, Argentina.

Cibils, A. F., and M. B. Coughenour. 2001. Impact of grazing management on the productivity of cold temperate grasslands of Southern Patagonia-a critical assessment. International Grassland Congress 2001. Sao Pedro, Sao Paulo, Brasil. Pp. 807-811.

Cingolani, A. M., I. Noy-Meir, D. D. Renison, and M. Cabido. 2008. La ganadería extensiva, ¿es compatible con la conservación de la biodiversidad y de los suelos? Ecología austral 18:253-271.

Consorcio DHV. 1999. Desertificación en la Patagonia. Informe Principal. Consorcio DHV Consultants-Sweedforest. Buenos Aires, Argentina. Pp. 111.

Coronato, F. R. 2015. Ovejas, territorio y políticas públicas en la Patagonia. Revista Estudios del ISHiR 5:6-19.

Deregibus, V. A. 1988. Importancia de los pastizales naturales en la República Argentina: situación presente y futura. Revista Argentina de Producción Animal 8:67-78.

Distel, R. A. 2013. Manejo del pastoreo en pastizales de zonas áridas y semiáridas. Revista Argentina de Producción Animal 33:53-64.

Fariña, C. M., G. L. Siffredi, M. Oesterheld, A. F. Cibils, and P. M. Willems. 2018. Pastoreo intensivo en distintas estaciones del año: efectos a escala de comunidad en una estepa de Patagonia Norte. VIII Congreso Nacional - IV Congreso del Mercosur sobre Manejo de Pastizales Naturales. Pp. 80.

Fuls, E. R. 1992. Semi-arid and arid rangelands: a resource under siege due to patch-selective grazing. Journal of Arid Environments 22:191-193. https://doi.org/10.1016/S0140-1963(18)30591-3

Golluscio, R. A., V. A. Deregibus, and J. M. Paruelo. 1998. Sustainability and range management in the Patagonian steppes. Ecología Austral 8:265-284.

Golluscio, R., C. Giraudo, P. Borrelli, L. Montes, G. Siffredi, G. Cechi, V. Nakamatsu, and J. Escobar. 1999. Utilización de los Recursos Naturales en la Patagonia. In: Consorcio DHV, Informe Técnico. Pp. 80.

González, L., and P. Rial. 2006. Ordenamiento base de datos catastro rural provincia de Santa Cruz. Convenio CFIDirección Provincial de Catastro-EEA Santa Cruz.

Hart, R. H., J. Bissio, M. J. Samuel, and J. W. Jr. Waggoner. 1993. Grazing systems, pasture size, and cattle grazing behavior and gains. Journal of Range Management 46:81-87. https:/ / doi.org/10.2307/4002452

Holechek, J. L., R. D. Pieper, and C. H. Herbel. 2010. Range management: principles and practices. 6th ed. PrenticeHall, Nueva Jersey, Estados Unidos. Pp. 456.

Hunt, L. P., S. Petty, R. Cowley, A. Fisher, A. J. Ash, and N. MacDonald. 2007. Factors affecting the management of cattle grazing distribution in northern Australia: preliminary observations on the effect of paddock size and water points. The Rangeland Journal 29:169-179. https://doi.org/10.1071/RJ07029

Iglesias, R., A. Schorr, M. Villa, and A. Vozzi. 2015. Situación actual y perspectiva de la ganadería en Patagonia Sur. Centro Regional Patagonia Sur. Instituto Nacional de Tecnología Agropecuaria. Pp. 24.

Martinic, M. 1985. La ocupación y el impacto del hombre sobre el territorio. Pp. 81-94 in O. Boelcke, D. Moore and F. Roig (eds.). Transecta botánica de la Patagonia Austral. CONICET, Instituto de la Patagonia, Royal Society, Buenos Aires, Argentina.

Milicevic, F. 2013. Diagnóstico del proceso de desarrollo territorial. Sustentabilidad social de la actividad ovina en la estepa magallánica del sur de Santa Cruz. Trabajo integrador para optar al grado de Especialista en Desarrollo Rural. 
Escuela para Graduados Alberto Soriano. Universidad de Buenos Aires. Buenos Aires, Argentina. Pp. 48.

Oliva, G., I. Noy-Meir, and A. Cibils. 2001. Fundamentos de ecología de pastizales. Pp. 83-100 in P. Borrelli and G. Oliva (eds.). Ganadería ovina sustentable en la Patagonia Austral. INTA, Buenos Aires, Argentina.

Oliva, G., D. Ferrante, S. Puig, and M. Williams. 2012. Sustainable sheep management using continuous grazing and variable stocking rates in Patagonia: a case study. The Rangeland Journal 34:285-295. https://doi.org/10.1071/ RJ12016

Oñatibia, G. R., and M. R. Aguiar. 2018. Paddock Size Mediates the Heterogeneity of Grazing Impacts on Vegetation. Rangeland Ecology and Management. https://doi.org/10.1016/j.rama.2018.03.002

Ormaechea, S. G., P. L. Peri, R. Molina, and J. P. Mayo. 2009. Situación y manejo actual del sector ganadero en establecimientos con bosque de ñire (Nothofagus antarctica) de Patagonia sur. 1er Congreso Nacional Silvopastoril. Posadas, Argentina. Pp. 385-393.

Ormaechea, S., and P. Peri. 2015. Landscape heterogeneity influences on sheep habits under extensive grazing management in Southern Patagonia. Livestock Research for Rural Development 27(6) art. 105.

Quargnolo, E., E. Carabelli, L. González, D. Suárez, C. Amicone, M. V. Sturzembaum, and E. Rivera. 2007. Determinación de la "brecha tecnológica" existente en los sistemas de producción ovina de la Patagonia austral, identificación de los puntos críticos e impacto económico, social y ambiental de la aplicación de la tecnología disponible recomendada para el manejo extensivo en el extremo sur de Santa Cruz. Informe técnico EEA INTA Santa Cruz. Río Gallegos, Argentina. Pp. 74.

Rimoldi, P. 2004. Producción ganadera sustentable en el árido chubutense. Pp. 36-40 in IDIA XXI Ovinos. Ediciones INTA, Buenos Aires, Argentina.

Schlesinger, W. H., J. F. Reynolds, G. L. Cunningham, L. F. Huenneke, W. M. Jarrell, R. A. Virginia, and W. G. Whitford. 1990. Biological feedbacks in global desertification. Science 247:1043-1048. https://doi.org/10.1126/ science.247.4946.1043

Sevi, A., A. Muscio, D. Dantone, V. Iascone, and F. D'Emilio. 2001. Paddock shape effects on grazing behavior and efficiency in sheep. Journal of Range Management 54:122-125. https://doi.org/10.2307/4003171

Soriano, A. 1983. Deserts and semideserts of Patagonia. Pp. 423-60 in N. E. West (ed.). Ecosystems of the World: Temperate Deserts and Semideserts, Elsevier, Amsterdam, the Netherlands.

Squires, V. R., A. D. Wilson, and G. T. Daws. 1972. Comparisons of the walking activity of some Australian sheep. Proceedings of the Australian Society of Animal Production 9:376-380.

Sturzenbaum, M. V. 2012. Los productores ganaderos ovino-extensivos y la adopción tecnológica en el sureste de la provincia de Santa Cruz. Trabajo integrador para optar al grado de Especialista en Desarrollo Rural. Escuela para Graduados Alberto Soriano. Universidad de Buenos Aires, Buenos Aires, Argentina. Pp. 83.

Suárez, D. 2009. Ovinos y alambres, paisaje cultural. Del pastizal natural a la insustentabilidad de la ganadería ovina en Santa Cruz. Tesis de Maestría en Desarrollo Sustentable. Convenio FLACAM - Universidad Nacional de Lanús, Argentina. Pp. 119.

Tanaka, J. A., N. R. Rimbey, L. A. Torell, D. T. Taylor, D. Bailey, T. DelCurto, K. Walburger, and B. Welling. 2007. Grazing distribution: the quest for the silver bullet. Rangelands 29:38-46. https:/ /doi.org/10.2458/azu_rangelands_ v29i4_tanaka

del Valle, H. F. 1998. Patagonian soils: a regional synthesis. Ecología Austral 8:103-122.

di Virgilio, A., and J. M. Morales. 2016. Towards evenly distributed grazing patterns: including social context in sheep management strategies. PeerJ 4:e2152. https://doi.org/10.7717/peerj.2152

Williams, R. E. 1954. Modern methods of getting uniform use of ranges. Journal of Range Management 7:77-81. https: //doi.org/10.2307/3893862

Williams, M. L. 2009. La situación del sector ovino en Santa Cruz: análisis y reflexiones. Estación Experimental Agropecuaria INTA Santa Cruz. Río Gallegos, Argentina. Pp. 27.

Woolfolk, E. J. 1955. Range improvement and management problems in Argentina. Journal of Range Management 8: 260-264. https://doi.org/10.2307/3893750 